\title{
Mitteilungen
}

\section{Deutsche Gesellschaft für Kieferorthopädie (e. V.)}

\section{„Amold-Biber-Preis 1978“}

Der Arnold-Biber-Preis wird hiermit für das Jahr 1978 ausgeschrieben. Für die Verleihung des Preises gilt das nachfolgende Statut:

\section{Präambel}

Die Firme ,,Dentaurum Pforzheim": hat einen nach dem Gründer ihrer Firma benannten ,ArnoldBiber-Preis" in Höhe von DM 4.000,- gestiftet, der von einem Kuratorium alljährlich vergeben werden soll.

Für die Zuerkennung des „Arnold-Biber-Preises" gilt folgendes Statut:

1. Der ,Arnold-Biber-Preis" wird für eine bisher noch nicht veröffentlichte wissenschaftliche Arbeit aus dem Gebiet der Kieferorthopädie verliehen.

2. Die Bewertung einer Arbeit erfolgt durch ein Kuratorium, das aus drei von der Deutschen Gesellschaft für Kieferorthopädie bestimmten Mitgliedern und dem Stifter des Preises oder seines Vertreters besteht. Der Vertreter der Firma Dentaurum enthält sich auf eigenen Wunsch eines Urteils über die wissenschaftliche Arbeit.

3. Um den Preis können sich in Deutschland approbierte Zahnärzte und die Mitglieder der Deutschen Gesellschaft für Kieferorthopädie bewerben.

4. Die Arbeit ist in vier Exemplaren in deutscher Sprache durckfertig bis spätestens 15. Februar 1979 dem 1. Vorsitzenden der Deutschen Gesellschaft für Kieferorthopädie unter Einschreiben einzureichen. Später eingereichte Arbeiten können für das laufende Jahr nicht mehr angenommen werden.

5. Die Arbeit darf den Verfasser nicht erkennen lassen, sie ist daher mit einem Kennwort zu versehen. Der Arbeit ist ein verschlossener Briefumschlag beizufügen, der das Kennwort trägt und den Namen und die Anschrift des Verfassers enthält.

6. Die Arbeit muß geistiges Eigentum des Bewerbers sein. Eine entsprechende Erklärung ist der Arbeit in dem verschlossenen Umschlag beizufügen.

7. Die Entscheidung des Kuratoriums ist endgültig, sie wird schriftlich dem 1. Vorsitzenden der Deutschen Gesellschaft für Kieferorthopädie mitgeteilt. Die Verleihung des Preises erfolgt anläßlich der wissenschaftlichen Jahrestagung der Deutschen Gesellschaft für Kieferorthopädie.

8. Über die Veröffentlichung der preisgekrönten Arbeit entscheidet das Kuratorium. Ansprüche auf Patente oder Musterschutz oder der nachträgliche Anspruch auf Verwertung der angegebenen Apparate usw. verbleiben beim Preistrăger.

9. Die nicht prämiierten Arbeiten werden den Absendern mittels Einschreibebrief zurückgegeben. Eine Teilung des Preises soll nicht erfolgen.

10. Wird von einem Bewerber bei der Einreichung der Arbeit gegen die Bestimmungen des Statuts verstoßen, so scheidet diese Arbeit aus der Bewerbung aus.

E. Housser, l. Vorsitzender 